\title{
Downregulation of $d r s$ mRNA expression is associated with the progression of adult T-cell leukemia/lymphoma
}

\author{
MISUZU SHIMAKAGE ${ }^{1,10}$, NOBUMASA INOUE ${ }^{2}$, KOHICHI OHSHIMA ${ }^{3}$, KUNIMITSU KAWAHARA $^{4}$, \\ NAOKI YAMAMOTO ${ }^{5}$, TAKASHI OKA ${ }^{6}$, YUKIHIRO TAMBE ${ }^{7}$, KAZUTA YASUI $^{8}$, \\ KAYOKO MATSUMOTO ${ }^{8}$, MASUO YUTSUDO ${ }^{9}$ and HIROKAZU INOUE ${ }^{7}$
}

\begin{abstract}
${ }^{1}$ Clinical Research Institute, ${ }^{2}$ Internal Medicine, Osaka National Hospital 2-1-14 Hoenzaka, Chuo-ku, Osaka, Osaka 540-0006; ${ }^{3}$ Department of Pathology, Faculty of Medicine, Kurume University, 67 Asahi-machi, Kurume, Fukuoka 830-0011; ${ }^{4}$ Department of Pathology, Osaka Prefectural Medical Center for Respiratory and Allergic Diseases, Habikino, Osaka 583-8588; ${ }^{5}$ Department of Microbiology, Medical Research Division, Tokyo Medical and Dental University, 1-5-45 Yushima, Bunkyo-ku, Tokyo 113-8519; ${ }^{6}$ Department of Pathology, Graduate School of Medicine and Dentistry, Okayama University, 2-5-1 Shikata-cho, Okayama, Okayama 700-8558;

${ }^{7}$ Department of Microbiology, School of Medicine, Shiga University of Medical Science, Setasukiwa-cho, Ohtsu, Shiga 520-2192; ${ }^{8}$ Red Cross Blood Center, 2-4-43 Morinomiya, Joto-ku, Osaka, Osaka 536-8505; ${ }^{9}$ Department of Cancer Cells, Research Institute for Microbial Diseases, Osaka University, 3-1 Yamadaoka, Suita, Osaka 565-0871, Japan
\end{abstract}

Received January 8, 2007; Accepted February 20, 2007

\begin{abstract}
Although adult T-cell leukemia/lymphoma (ATLL) is initiated by infection with human T-cell leukemia virus (HTLV-1), many other factors are thought to be required for the progression from indolent ATL to aggressive ATLL. The $d r s$ gene was originally isolated as a novel suppressor gene of v-src transformation and was shown to induce apoptosis in human cancer cells. To investigate the involvement of $d r s$ downregulation in the progression of ATLL, we examined the expression of $d r s$ in smoldering, chronic and aggressive ATLL, and found that $d r s$ expression was markedly reduced in clinically aggressive ATLL. In aggressive ATLL cell lines, expression of $d r s$ mRNA was not detected, although expression of $d r s$ mRNA was detected in T-cell lines infected with HTLV-1. A correlation between $d r s$ downregulation and expression of the Tax gene was not observed in these T-cell lines. Furthermore, introduction of $d r s$ into an ATL cell line, HUT102, by retrovirus vector suppressed the colony formation of the cells in soft agar and enhanced apoptotic cell death of
\end{abstract}

Correspondence to: Dr Hirokazu Inoue, Department of Microbiology, School of Medicine, Shiga University of Medical Science, Setasukiwa-cho, Ohtsu, Shiga 520-2192, Japan

E-mail: hirokazu@belle.shiga-med.ac.jp

${ }^{10}$ Present address: Department of Pediatrics, National Hospital Organization, Wakayama National Hospital, 1138 Wada, Mihamacho, Hidaka-gun, Wakayama 644-0044, Japan

Key words: drs, adult T-cell leukemia/lymphoma, progression the cells under low serum culture conditions. These results indicate that downregulation of $d r s$ is closely linked to the progression of ATLL, independently of Tax expression, suggesting that $d r s$ may suppress the progression of ATLL via enhancing apoptosis.

\section{Introduction}

Adult T-cell leukemia/lymphoma (ATLL) is an aggressive T-cell malignancy in humans. ATLL develops in $2-6 \%$ of people infected with human T-cell leukemia virus type 1 (HTLV-1), after a long latency of up to 20-50 years $(1,2)$. ATLL is divided into four subtypes or stages: the smoldering, chronic, lymphoma and acute types. The smoldering and chronic types are indolent, but the acute and lymphoma types are aggressive ATLLs, characterized by resistance to chemotherapy and a poor prognosis (3). HTLV-1 has been shown to be a causative agent of ATLL. The viral protein Tax has been proposed to play a crucial role in the genesis of ATLL, because of its transforming activity in various experimental systems, including primary human T-cells and rodent fibroblast cells (4-6). The Tax protein promotes the transcriptional activation of a specific set of cellular genes, trans-repression of another set of genes and cell cycle progression through the inactivation of tumor suppressor proteins (6). However, the mechanism of the progression from indolent types of ATL to acute ATLL remains to be elucidated.

The $d r s$ gene was originally isolated as a suppressor gene against $v$-src transformation $(7,8)$. Expression of $d r s$ mRNA was markedly reduced in a variety of human cancer cell lines and malignant tumor tissues, including those of the colon, bladder, ovary, lung, and prostate (9-12). Introduction of $d r s$ cDNA by retrovirus vector into these cancer cell lines suppressed anchorage-independent growth (9). Malignant 
tumors including T-cell lymphomas, lung adenocarcinomas, and hepatomas were generated in approximately $30 \%$ of the $d r s$ knockout (KO) mice, indicating that $d r s$ contributes to the suppression of malignant tumor formation (13). We have also found that the Drs protein interacted with ASY/ Nogo-B/RTN- ${ }_{S}$, an apoptosis-inducing protein localized in the endoplasmic reticulum, and ectopic expression of the Drs protein induced apoptosis associated with the activation of caspases in various human cancer cell lines (14). These findings suggest that the drs gene is involved in the suppression of tumor formation by promoting apoptosis. To clarify the correlation between downregulation of $d r s$ mRNA and the progression of ATLL, we compared the expression level of $d r s$ mRNA in advanced and indolent ATLL specimens by in situ hybridization and examined it in ATL cell lines by RT-PCR. Furthermore, we investigated the effect of introduction of $d r s$ into an ATL cell line by retrovirus vector.

\section{Materials and methods}

Patients. Thirty-three patients with ATLL were selected from the ATLL files (1998-2002) of Fukuoka University and Osaka National Hospital. Peripheral blood samples of all cases were assumed to contain HTLV-1 provirus by PCR. None of these cases had features indicative of an immunocompromised state. The ATLL patients included 15 females and 18 males, with a mean age of 62.7 years. The clinical classification was in accordance with the general rules indicated in a previously reported literature (3). Approximately $1-5 \%$ of peripheral blood ATL cells were morphologically abnormal, and the percentage was nearly the same in smoldering, chronic and acute ATL. Normal peripheral blood, normal T-cells (CD4+, $\left.\mathrm{CD}^{+}\right)$were separated from healthy adult blood donors who visited the Red Cross Blood Center, Osaka.

Cell lines. The cell lines used in this study were human Tcells transformed with HTLV-1, MT-2 and MT-4, and those established from aggressive ATLL, HUT102 and TL-OmI. Jurkat and Molt-4 (non-ATL T-cell lymphoma lines) and WI38 (normal human fibroblasts) were also used as positive controls for the expression of $d r s$ mRNA. These cells were cultured in RPMI-1640 medium supplemented with $10 \%$ fetal calf serum (FCS).

In situ hybridization. Formalin-fixed paraffin-embedded lymphoma tissues were cut into serial sections of $3-\mu \mathrm{m}$ thickness. Spreading samples of peripheral blood were fixed with $4 \%$ paraformaldehyde in phosphate buffered saline (PBS) (pH 7.0) overnight at $4^{\circ} \mathrm{C}$. As a probe, human $d r s$ cDNA was cloned into the plasmid, pBluescript II SK(-). The antisense and sense RNA probes were labelled with digoxigenin-11-UTP by in vitro transcription using a commercial kit (RocheBoehringer-Mannheim, Indianapolis, IN). The labelled riboprobes were then fragmented to approximately 100 bases in length by alkaline hydrolysis. The sense probe served as a negative control. In situ hybridization was performed as reported previously $(10,14,15)$. The adjacent sections of the paraffin blocks were stained with hematoxylin and eosin (H\&E). Each one of the spreading peripheral blood samples was stained with Giemsa.
Northern blot analysis. Isolation of total RNA, electrophoresis, blotting and hybridization were performed as previously described (9).

$R T$-PCR. Reverse transcription was carried out using total RNAs $(2 \mu \mathrm{g})$ from the cells. Oligo(dT)-adaptor primers (Takara, Tokyo) were used for the reverse transcription reaction. The reverse transcription products were amplified by 30 cycles using degenerate primers $(0.4 \mu \mathrm{M})$, Takara Ex Taq polymerase (2.5 units). The primer sets used for PCR in order to detect human $d r s$ mRNA were 5'-TTAAGTGAGCT GTGCAGCCT-3' for the forward primer (pDrsU2), and 5'TAACAGCACATCAGACGTTGC-3' for the reverse primer (pDrsSR3, this is located in the $3^{\prime}$ downstream of the consensus repeat 2 of $d r s$ ). The primer sets used to detect Tax of HTLV-I were 5'-AGGGTTTGGACAGAGTCTT-3' for the forward primer and 5'-GTAAGGACCTTGAGGGTCTTAG-3' for the reverse primer. Amplicons were separated on 1.5\% agarose gel.

Soft agar and cell proliferation assay. For soft agar assay, $1 \times 10^{5}$ cells were inoculated onto $0.4 \%$ soft agar containing RPMI medium supplemented with $10 \%$ FCS. The number of colonies per dish was scored at 3 weeks after inoculation. For cell proliferation assay, $2 \times 10^{5}$ cells were inoculated into a 35-mm dish containing $2 \mathrm{ml}$ of RPMI medium supplemented with $10 \%, 3 \%$, or $1 \%$ FCS. Culture medium (1 ml) was replaced with fresh medium every 3 days. The number of viable cells per dish was scored at 12 days after inoculation and quantified by trypan blue staining.

Apoptosis assay. The cell culture was performed in a $35-\mathrm{mm}$ dish containing $2 \mathrm{ml}$ of RPMI medium supplemented with $10 \%$ or $1 \%$ FCS as described in cell proliferation assay. The characterization and quantification of apoptotic cell death was performed using Annexin-PI assay as previously described (14).

\section{Results}

Downregulation of drs mRNA in lymphoma tissues of ATL patients. To clarify the correlation between expression of the $d r s$ gene and progression of ATLL, we examined seventeen cases of ATLL lymphoma for the expression of the drs gene using mRNA in situ hybridization. Fifteen cases showed no signals with the antisense $d r s$ probe (Fig. 1A), although two cases showed weak signals (Table I). On the other hand, in tissues of non-ATLL T-cell lymphoma, positive signals were detected by the anti-sense probe, but not by the sense probe in all seven cases examined (Fig. 1B and Table I). drs mRNA was also expressed in tissues of rheumatoid arthritis (RA), Castleman's disease (Fig. 1B) and reactive lymphoproliferation (Table I). In normal T-cells $\left(\mathrm{CD}^{+}\right.$and $\left.\mathrm{CD}^{+}\right)$and the nonATLL T-cell lines, Jurkat and Molt-4, expression of $d r s$ mRNA was detected by in situ hybridization (Fig. 2A and B and Table I). These results, which are summarized in Table I, indicate that $d r s$ expression was suppressed specifically in ATL lymphomas.

Downregulation of drs $m R N A$ in peripheral blood of aggressive ATLL patients. To further confirm that downregulation of $d r s$ 
A

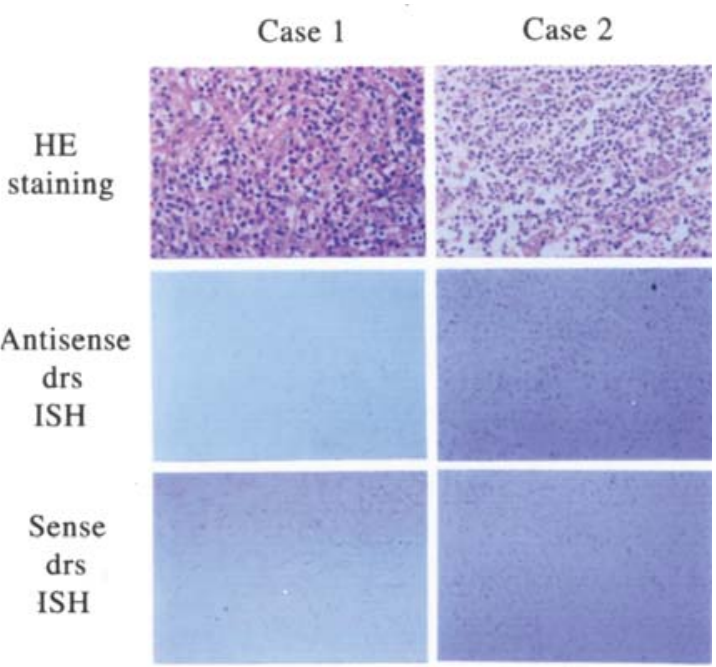

B

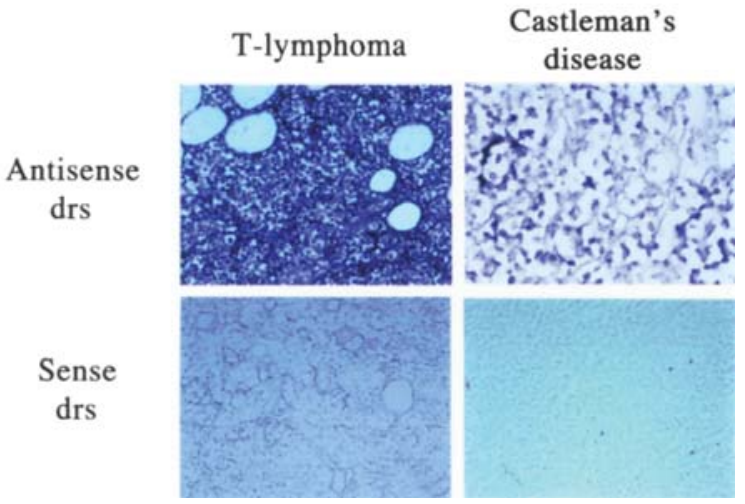

Figure 1. Expression of $d r s$ mRNA in lymphoma tissues of ATL patients (case 1 and 2) (A) and in a case of non-ATL T-cell lymphoma (case 1) and a case of Castleman's disease (B). Original magnification x200.

\section{Peripheral blood}

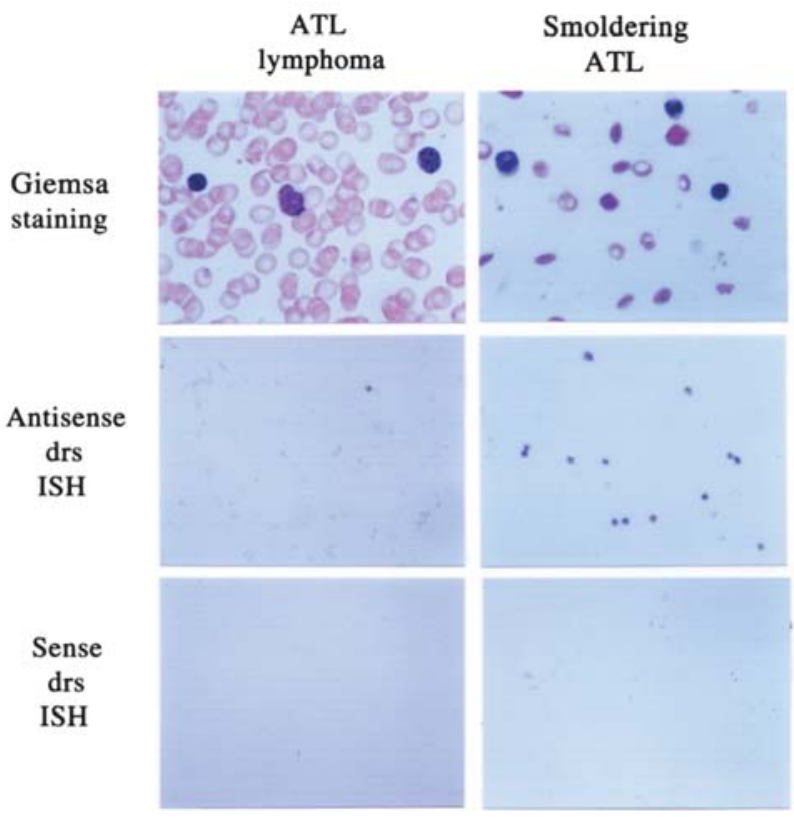

Figure 3. Expression of drs mRNA in the peripheral blood of ATL patients. mRNA in situ hybridization was performed as described in the Materials and methods. Right, smoldering ATL; left, ATL lymphoma.
A

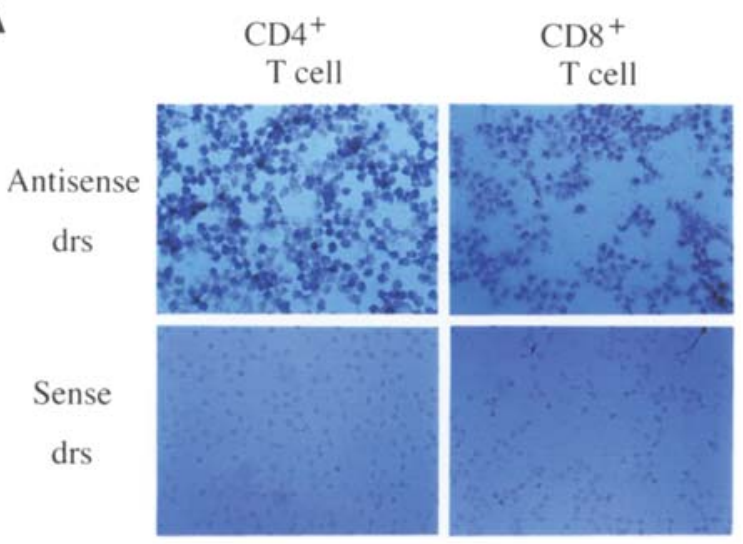

B

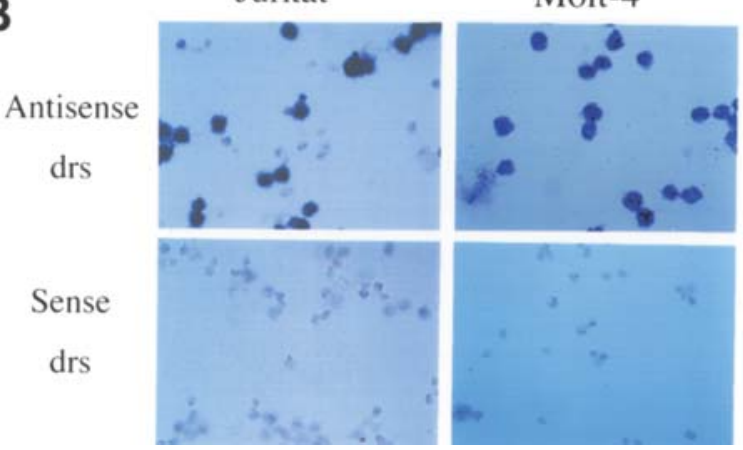

Figure 2. Expression of $d r s$ mRNA in normal human T cells (A) (left, CD4+; right, $\mathrm{CD}^{+}$) and non-ATL T-cell lines, Jurkat and Molt-4 (B). Original magnification $\times 200$.

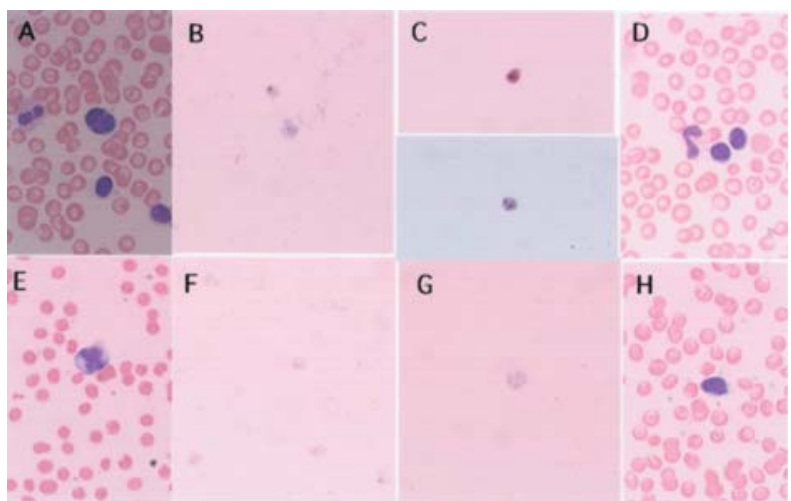

Figure 4. Expression of drs mRNA by in situ hybridization in peripheral blood of acute ATL patients before and after chemotherapy. Case 7: A and B, before treatment; $\mathrm{C}$ and $\mathrm{D}$, remitted after treatment. Case 4: $\mathrm{E}$ and $\mathrm{F}$, before treatment; $\mathrm{G}$ and $\mathrm{H}$, not remitted. A, D, E, and H, Giemsa staining. B, C, F, and $\mathrm{G}, d r s$ mRNA by in situ hybridization.

correlates with the progression of ATLL, we examined the expression of $d r s$ mRNA in the peripheral blood of smoldering, chronic and acute ATLL patients by drs mRNA in situ hybridization. Three of six smoldering ATL cases showed positive hybridization signals, although these contained many atypical large lymphocytes (Fig. 3 right panel and Table I). In one of the three chronic ATL cases, a positive signal was observed (Table I), whereas no signals were detected in the 
Table I. Expression of $d r s$ mRNA by in situ hybridization.

\begin{tabular}{ccccc}
\hline & Case & Age & Sex & $d r s$ \\
\hline ATL lymphoma & 1 & 68 & F & - \\
& 2 & 74 & M & - \\
& 3 & 57 & M & - \\
4 & 63 & M & - \\
& 5 & 77 & M & - \\
& 6 & 65 & F & - \\
7 & 70 & M & - \\
& 8 & 82 & F & - \\
& 9 & 82 & F & - \\
& 10 & 57 & F & - \\
& 11 & 68 & F & - \\
& 12 & 61 & F & \pm \\
& 13 & 70 & F & - \\
& 14 & 66 & F & - \\
& 15 & 65 & M & - \\
& 16 & 50 & M & \pm \\
& 17 & 63 & M & - \\
& 1 & 39 & M & + \\
& 2 & 38 & M & \pm \\
& 3 & 76 & F & + \\
& 4 & 45 & F & w+ \\
& 5 & 56 & F & ++ \\
6 & 44 & F & ++ \\
& 7 & 45 & F & ++
\end{tabular}

Controls

RA

Castleman's disease

Reactive

1

2

3

4

5

Peripheral blood

Smoldering

Chronic

Acute
Table I. Continued.

\begin{tabular}{|c|c|c|c|c|}
\hline & Case & Age & Sex & $d r s$ \\
\hline \multicolumn{5}{|l|}{ Controls } \\
\hline Normal T, CD4+ & & & & ++ \\
\hline Normal T, $\mathrm{CD}^{+}$ & & & & $\mathrm{w}+$ \\
\hline Normal B, CD19L & & & & $\mathrm{w}+$ \\
\hline Molt-4 & & & & ++ \\
\hline Jurkat & & & & ++ \\
\hline
\end{tabular}

w, weakly; \pm , equivocal; RA, rheumatoid arthritis; reactive, reactive lymphoproliferation.

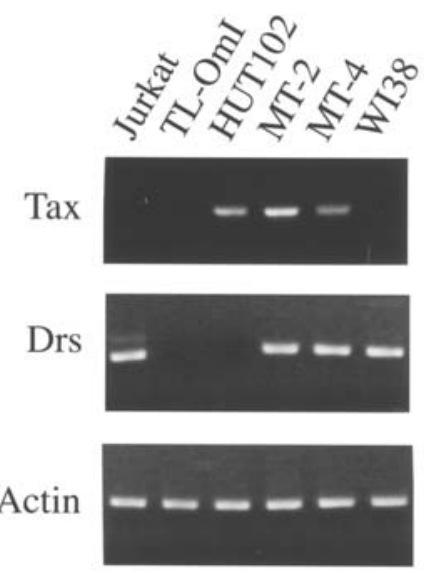

Figure 5. RT-PCR analysis for the expression of $d r s$ and HTLV-1 Tax mRNA in ATL and non-ATL T-cell lines. The expression of $\beta$-actin was used as an internal control.

seven cases of acute ATLL (Fig. 3 left panel and Table I). These results indicate that the expression of the $d r s$ gene is suppressed along with progression of ATLL. Furthermore, we followed four patients with acute ATL for 2.5-13 months after chemotherapy. Three of four patients were remitted by the chemotherapy and one patient was still in the course of treatment. Fig. 4 shows the results of one (acute case 7) of the remitted cases and the single not-remitted case (acute case 4). At the beginning of treatment, all cases showed a serious decreased number of lymphocytes in the peripheral blood. Although the morphology of lymphocytes did not change (Fig. 4A, D, E, and H), the remitted case (Fig. 4C) showed significantly stronger $d r s$-positive signals compared with the non-remitted case (Fig. 4G) and non-treated cases (Fig. 4B and F). The increased intensity of signals by in situ hybridization correlated with the clinical effectiveness of the treatment. These results indicate that the expression of the $d r s$ gene coincides with the degree of recovery in progressed ATLL.

Expression of drs mRNA in ATL cell lines. We also examined the expression of $d r s$ mRNA by RT-PCR in T-cell lines established from aggressive ATL patients, HUT102 and TL-OmI, and T-cell lines infected with HTLV-1, MT-2 and MT-4. A non-ATL T-cell line, Jurkat, and human fibroblasts, 
A

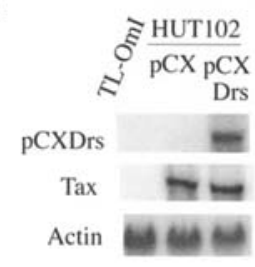

B

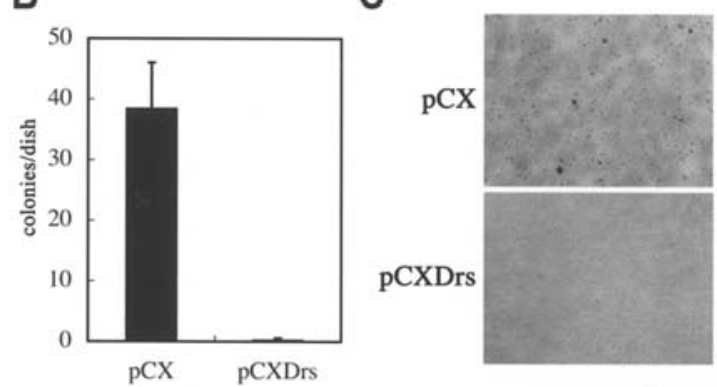

Figure 6. Effects of ectopic expression of the drs gene in HUT102. A, expression of exogenous drs mRNA in HUT102 infected with a retrovirus containing the $d r s$ gene. $\mathrm{B}$, colony formation in soft agar. C, colony formation on soft agar.
A

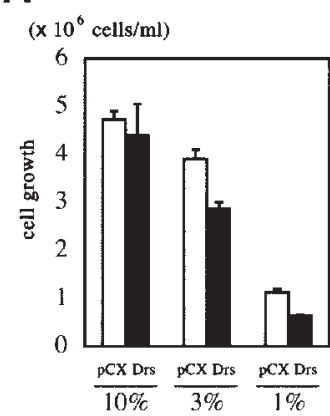

B

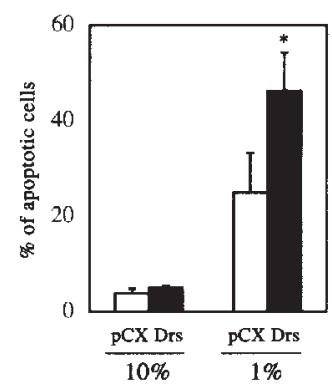

Figure 7. Cell proliferation (A) and apoptotic cell death (B) under high and low serum culture conditions. Cells $\left(2 \times 10^{5}\right)$ were inoculated into a $35-\mathrm{mm}$ dish containing $2 \mathrm{ml}$ of RPMI medium supplemented with $10 \%, 3 \%$, or $1 \%$ FCS. ${ }^{*} \mathrm{P}<0.05$ by Welch's t-test.

WI38, were used as positive controls. As shown in Fig. 5, the expression of $d r s$ mRNA was completely downregulated in two ATL cell lines (HUT102 and TL-OmI), but significantly expressed in the non-ATL T-cell lines (MT-2, MT-4, and Jurkat) and fibroblast cells (WI38). Expression of HTLV-1 mRNA encoding the Tax gene was also examined by RT-PCR. Although Tax mRNA was expressed in an ATL cell line, HUT102, and two cell lines infected with HTLV-1, it was not detected in another ATL cell line, TL-OmI (Fig. 5), indicating that downregulation of $d r s$ mRNA is not dependent on expression of the Tax gene of HTLV-1.

Suppression of anchorage-independent growth and enhancement of apoptotic cell death of an ATL cell line, HUT102, by the drs gene. To investigate whether the drs gene can suppress the growth properties of ATL cells, we introduced the $d r s$ gene into an HUT102 cell line by retrovirus vector. HUT102 cells were infected with a recombinant retrovirus containing $d r s$ (pCXdrs) (13) and a vector virus (pCX) (16). The blasticidin-resistant cells were collected and assayed for colony formation in soft agar and cell proliferation under high and low serum culture conditions. The expression of viral mRNA containing the $d r s$ gene was confirmed by Northern blot analysis (Fig. 6A). The expression of Tax mRNA of HTLV-1 in HUT102-pCXdrs cells was similar to that in HUT102-pCX cells (Fig. 6A). As shown in Fig. 6B and C, a significant number of colonies in soft agar were detected in HUT102-pCX cells, although the efficiency of colony formation was low. On the other hand, colonies were scarcely observed in HUT102-pCXdrs cells. As shown in Fig. 7A, the population of viable cells of HUT102-pCXdrs cells under low serum (1\% and $3 \%)$ culture conditions was decreased compared with that of HUT102-pCX. On the other hand, the percentage of apoptotic cells was increased in HUT102pCXdrs cells, as compared to that of HUT102-pCX cells, under low serum (1\%) culture conditions (Fig. 7B). These results indicate that ectopic expression of drs is able to suppress the growth properties and enhance apoptotic cell death of HUT102 ATL cells.

\section{Discussion}

In the present study, we showed that $d r s$ expression was markedly reduced in lymphoma tissues of aggressive ATL patients and T-cell lines, HUT102 and TL-OmI, derived from aggressive ATLL. Experiments using peripheral blood of smoldering, chronic and acute ATL patients also showed that downregulation of $d r s$ mRNA is associated with aggressive ATL. The $d r s$ expression was restored in peripheral lymphocytes of ATLL patients who were remitted by chemotherapy. In addition to these results, we also found that introduction of drs into HUT102 cells by retrovirus vector caused suppression of colony formation in soft agar and enhancement of apoptotic cell death under low serum culture conditions. These findings demonstrated a significant association between the downregulation of $d r s$ mRNA and progression of ATLL, suggesting that $d r s$ may suppress ATLL progression.

The progression of ATLL has been extensively investigated and cellular factors other than HTLV-1 Tax expression have been proposed to be involved in the onset or progression of ATLL. Stochastic analyses support the notion that there are more than 5 steps in the process of ATLL progression (17). TP53 gene mutation and the loss of heterozygosity (18) and alteration (19) or methylation (20) of the p16 (CDKN2A) gene have been reported in the course of ATLL. The expression and phosphorylation of the $\mathrm{Rb}$ protein $(21)$ and the activation

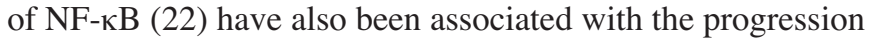
of ATLL. However, the key molecules triggering the onset or progression of ATLL have not been determined. In this study, we proposed that the $d r s$ gene plays a critical role for the progression of ATL, because the expression of this gene was markedly suppressed in ATL lymphomas, almost all cases of advanced ATLL, and T-cell lines derived from aggressive ATL, while drs mRNA was expressed in $50 \%$ of smoldering cases, the less malignant type of ATLL, and T-cell lines infected with HTLV-1. Interestingly, T-cell lymphomas were 
generated in six (13\%) of 46 drs KO mice (13), suggesting that loss of $d r s$ expression is critical for the development of T-cell lymphoma. Previously, we have shown that $d r s$ induces apoptosis mediated by caspase-3, -9 and -12 in human cancer cell lines (14), suggesting that its downregulation may lead to evasion of apoptosis. Reintroduction of $d r s$ into a lung cancer cell line derived from the tumor of a $d r s \mathrm{KO}$ mouse led to the suppression of tumor formation in nude mice, which was accompanied by enhanced apoptosis and enhanced sensitivity to apoptosis mediated by caspase-3, -9 and -12 under low serum culture conditions (13). Evasion of apoptosis has been shown to be essential for the malignant progression of various tumors, including leukemias and lymphomas $(23,24)$. Downregulation of the $d r s$ gene may cause progression of ATLL, at least in part, by protecting cells from apoptosis. In fact, ectopic expression of $d r s$ in an ATL cell line, HUT102, caused enhancement of apoptotic cell death under low serum culture conditions as shown in Fig. 7B. Recently, we also found that transcription of ASY, which is associated with Drs and induces apoptosis synergistically with $d r s$ (14), is also downregulated in aggressive ATLL (25). Downregulation of both apoptosisinducing genes may be critical for the progression of ATLL. From our previous studies on the functions of $d r s$ for human cancer cells $(9,14)$ and the present findings on the progression of ATLL, it is conceivable that the $d r s$ gene may be useful for the treatment of advanced ATLL. Introduction of the $d r s$ gene into hematopoietic stem cells may be effective for patients with acute ATLL, although further investigations will be required for clinical application.

\section{Acknowledgments}

We thank Dr R. Ueda (Nagoya City University) for his kind support of our study. We also thank Dr H. Nakamine (Nara Medical College) and Dr S. Takemoto (Kochi Medical University) for their generous help in this study. This study was supported in part by a Grant-in-Aid for Cancer Research (15-4) from the Ministry of Health, Labour and Welfare of Japan (to M. Shimakage). This project was also supported by a Grant-in-Aid for Scientific Research (C) (grant no. 15590335 and no. 17590341), and a Grant-in-Aid for Scientific Research on Priority Areas (C) (grant no. 16021222) from the Ministry of Education, Science, Sports, and Culture of Japan (to H. Inoue).

\section{References}

1. Poietz BJ, Ruscetti FW, Gazdar AF, Bunn PA, Minna JD and Gallo RC: Detection and isolation of type $C$ retrovirus particles from fresh and cultured lymphocytes of a patient with cutaneous T-cell lymphoma. Proc Natl Acad Sci USA 77: 7415-7419, 1980.

2. Uchiyama T, Yodoi J, Sagawa K, Takatsuki K and Uchino H: Adult T-cell leukemia: Clinical and hematologic features of 16 cases. Blood 50: 481-492, 1977.

3. Shimoyama M and Members of Lymphoma Study Group: Diagnostic criteria and classification of clinical subtypes of adult T-cell leukemia/lymphoma. Br J Haematol 79: 428-439, 1991.

4. Neuveut C, Low KG, Maldarelli F, Schmitt I, Majone F, Grassmann R and Jeang KT: Human T-cell leukemia virus type 1 Tax and cell cycle progression: role of cyclin D-cdk and p110RB. Mol Cell Biol 18: 3620-3632, 1998.
5. Pozzatti R, Vogel J and Jay G: The human T-lymphotropic virus type I tax gene can cooperate with the ras oncogene to induce neoplastic transformation of cells. Mol Cell Biol 10: 413-417, 1990.

6. Yoshida M: Multiple viral strategies of HTLV-1 for dysregulation of cell growth control. Annu Rev Immunol 19: 475-496, 2001.

7. Pan J, Nakanishi K, Yutsudo M, Inoue H, Li Q, Oka K, Yoshioka $\mathrm{N}$ and Hakura A: Isolation of a novel gene downregulated by v-src. FEBS Lett 383: 21-25, 1996.

8. Inoue H, Pan J and Hakura A: Suppression of v-src transformation by the $d r s$ gene. J Virol 72: 2532-2537, 1998

9. Yamashita A, Hakura A and Inoue H: Suppression of anchorageindependent growth of human cancer cell lines by the $d r s$ gene. Oncogene 18: 4777-4787, 1999.

10. Shimakage M, Kawahara K, Kikkawa N, Sasagawa T, Yutsudo M and Inoue H: Down-regulation of drs mRNA in human colon adenocarcinomas. Int J Cancer 87: 5-11, 2000.

11. Shimakage M, Takami K, Kodama K, Kodama K, Mano M, Yutsudo $\mathrm{M}$ and Inoue $\mathrm{H}$ : Expression of $d r s$ mRNA in human lung adenocarcinoma. Hum Pathol 33: 615-619, 2002.

12. Kim CJ, Shimakage M, Kushima R, Mukaisho K, Shinka T, Okada $\mathrm{Y}$ and Inoue H: Down-regulation of $d r s$ mRNA in human prostate carcinomas. Hum Pathol 34: 654-657, 2003.

13. Tambe Y, Yoshioka-Yamashita A, Mukaisho K, Haraguchi S, Chano T, Isono T, Kawai T, Suzuki Y, Kushima R, Hattori T, Goto M, Yamada S, Kiso M, Saga Y and Inoue H: Tumor prone phenotype of mice deficient in a novel apoptosis-inducing gene, drs. Carcinogenesis (in press).

14. Tambe Y, Isono T, Haraguchi S, Haraguchi S, YoshiokaYamashita A, Yutsudo M and Inoue H: A novel apoptotic pathway induced by the $d r s$ tumor suppressor gene. Oncogene 23: 2977-2987, 2004.

15. Shimakage M, Horii K, Tempaku A, Kakudo K, Shirasawa T and Sasagawa T: Association of Epstein-Barr virus with oral cancers. Hum Pathol 33: 608-614, 2002.

16. Akagi Y, Shishido T, Murata K and Hanafusa H: v-Crk activates the phosphoinositide 3-kinase/AKT pathway in transformation. Proc Natl Acad Sci USA 97: 7290-7295, 2000.

17. Okamoto T, Ohno Y, Tsugane S, Watanabe S, Shimoyama M, Tajima K, Miwa M and Shimotohno K: Multi-step carcinogenesis model for adult T-cell leukemia. Jpn J Cancer Res 80: 191-195, 1989.

18. Nishimura S, Asou N, Suzushima H, Okubo T, Fujimoto T, Osato M, Yamasaki H, Lisha L and Takatsuki K: p53 gene mutation and loss of heterozygosity are associated with increased risk of disease progression in adult $\mathrm{T}$ cell leukemia. Leukemia 9: 598-604, 1995.

19. Fujiwara H, Arima N, Hashimoto-Tamaoki T, Matsushima K, Ohtsubo H, Arimura K, Hidaka S and Tei C: Alteration of p16 (CDKN2) gene is associated with interleukin-2-induced tumor cell growth in adult T-cell leukemia. Exp Hematol 27: 1004-1009, 1999 .

20. Nosaka K, Maeda M, Tamiya S, Sakai T, Mitsuya H and Matsuoka M: Increasing methylation of the CDKN2A gene is associated with the progression of adult T-cell leukemia. Cancer Res 15: 1043-1048, 2000

21. Nakayama K, Yamada Y, Koji T, Hayashi T, Tomonaga M and Kamihira S: Expression and phosphorylation status of retinoblastoma protein in adult T-cell leukemia/lymphoma. Leuk Res 24: 299-305, 2000.

22. Arima N, Matsushita K, Obata H, Otsubo H, Fujiwara H, Arimura K, Kukita T, Suruga Y, Wakamatsu S, Hidaka S and Tei C: NF-kappaB involvement in the activation of primary adult T-cell leukemia cells and its clinical implications. Exp Hematol 27: 1168-1175, 1999.

23. Tsujimoto $\mathrm{Y}$, Ikegami $\mathrm{N}$ and Croce $\mathrm{CM}$ : Characterization of the protein product of bcl-2, the gene involved in human follicular lymphoma. Oncogene 2: 3-7, 1987.

24. Inukai $\mathrm{T}$, Inoue $A$, Kurosawa $\mathrm{H}$, Goi K, Shynjo $\mathrm{T}$, Ozawa $\mathrm{K}$, Mao M, Inaba T and Look AT: SLUG, a ces-1-related zinc finger transcription gene with antiapoptotic activity, is a downstream target of the E2A-HLF oncoprotein. Mol Cell 4: 343-352, 1999.

25. Shimakage M, Inoue N, Oshima S, Kawahara K, Oka T, Yasui G, Matsumoto K, Inoue H, Watari A, Higashiyama S and Yutsudo M: Down-regulation of ASY/Nogo transcription associated with progression of adult T-cell leukemia/lymphoma. Int J Cancer 119: 1648-1653, 2006. 